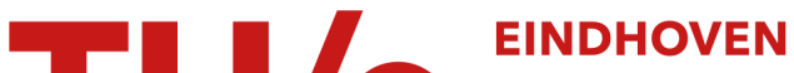

\section{Exciton bimolecular annihilation dynamics in supramolecular nanostructures of conjugated oligomers}

Citation for published version (APA):

Daniel, C., Herz, L. M., Silva, C., Hoeben, F. J. M., Jonkheijm, P., Schenning, A. P. H. J., \& Meijer, E. W. (2003). Exciton bimolecular annihilation dynamics in supramolecular nanostructures of conjugated oligomers. Physical Review B, 68(23), 235212-1/9. [235212]. https://doi.org/10.1103/PhysRevB.68.235212

DOI:

10.1103/PhysRevB.68.235212

Document status and date:

Published: 01/01/2003

\section{Document Version:}

Publisher's PDF, also known as Version of Record (includes final page, issue and volume numbers)

\section{Please check the document version of this publication:}

- A submitted manuscript is the version of the article upon submission and before peer-review. There can be important differences between the submitted version and the official published version of record. People interested in the research are advised to contact the author for the final version of the publication, or visit the $\mathrm{DOI}$ to the publisher's website.

- The final author version and the galley proof are versions of the publication after peer review.

- The final published version features the final layout of the paper including the volume, issue and page numbers.

Link to publication

\section{General rights}

Copyright and moral rights for the publications made accessible in the public portal are retained by the authors and/or other copyright owners and it is a condition of accessing publications that users recognise and abide by the legal requirements associated with these rights.

- Users may download and print one copy of any publication from the public portal for the purpose of private study or research.

- You may not further distribute the material or use it for any profit-making activity or commercial gain

- You may freely distribute the URL identifying the publication in the public portal.

If the publication is distributed under the terms of Article $25 \mathrm{fa}$ of the Dutch Copyright Act, indicated by the "Taverne" license above, please follow below link for the End User Agreement:

www.tue.nl/taverne

Take down policy

If you believe that this document breaches copyright please contact us at:

openaccess@tue.nl

providing details and we will investigate your claim. 


\title{
Exciton bimolecular annihilation dynamics in supramolecular nanostructures of conjugated oligomers
}

\author{
Clément Daniel, Laura M. Herz, and Carlos Silva* \\ Cavendish Laboratory, University of Cambridge, Madingley Road, Cambridge CB3 OHE, United Kingdom
}

Freek J. M. Hoeben, Pascal Jonkheijm, Albertus P. H. J. Schenning, and E. W. Meijer Laboratory of Macromolecular and Organic Chemistry, Eindhoven University of Technology, P.O. Box 513, 5600 MB Eindhoven, The Netherlands

(Received 16 July 2003; published 31 December 2003)

\begin{abstract}
We present femtosecond transient absorption measurements on $\pi$-conjugated supramolecular assemblies in a high-pump-fluence regime. Oligo( $p$-phenylenevinylene) monofunctionalized with ureido-s-triazine (MOPV) self-assembles into chiral stacks in dodecane solution below $75^{\circ} \mathrm{C}$ at a concentration of $4 \times 10^{-4} \mathrm{M}$. We observe exciton bimolecular annihilation in MOPV stacks at high excitation fluence, indicated by the fluencedependent decay of $1{ }^{1} B_{u}$-exciton spectral signatures and by the sublinear fluence dependence of time- and wavelength-integrated photoluminescence (PL) intensity. These two characteristics are much less pronounced in MOPV solution where the phase equilibrium is shifted significantly away from supramolecular assembly, slightly below the transition temperature. A mesoscopic rate-equation model is applied to extract the bimolecular annihilation rate constant from the excitation fluence dependence of transient absorption and PL signals. The results demonstrate that the bimolecular annihilation rate is very high with a square-root dependence in time. The exciton annihilation results from a combination of fast exciton diffusion and resonance energy transfer. The supramolecular nanostructures studied here have electronic properties that are intermediate between molecular aggregates and polymeric semiconductors.
\end{abstract}

DOI: 10.1103/PhysRevB.68.235212

PACS number(s): 78.47.+p, 78.55.Kz, 78.67.-n

\section{INTRODUCTION}

Organic semiconductor materials are now widely used in optoelectronics devices such as field-effect transistors, ${ }^{1,2}$ light-emitting diodes, ${ }^{3,4}$ and photovoltaic diodes ${ }^{5-7}$ Intramolecular functionality ( $\pi$ conjugation) can be tailored with synthetic methodologies, ${ }^{8-10}$ but control of intermolecular properties, which are equally important in determining electronic properties (e.g., charge transport), is more elusive. Perhaps the most compelling advantage of many organic semiconductors such as conjugated polymers over molecular and inorganic semiconductors is solution processability, making fabrication of elegantly simple device structures possible with techniques such as ink-jet printing. ${ }^{11,12}$ A natural strategy to achieve three-dimensional control of intermolecular interactions is to exploit molecular self-assembly in solution prior to the casting process by means of supramolecular chemistry. ${ }^{13}$ With this approach, molecular building blocks are self-assembled to form well-defined, complex architectures through secondary forces, such as electrophilicelectrophobic interactions. These interactions need to be strong enough to lead to spontaneous self-organization but weak enough so that the process is reversible. Supramolecular assemblies possess polymeric characteristics and are therefore often referred to as "supramolecular polymers." Their macroscopic properties can be tuned such that high carrier mobilities, for example, can be achieved. ${ }^{14}$

Recently, this approach has been applied very successfully to oligo( $p$-phenylenevinylene) derivatives with chiral side chains and functionalized with ureido-s-triazine, a hydrogen-bonding end group. ${ }^{15,16}$ It has been shown that these monofunctionalized oligo( $p$-phenylenevinylene) (MOPV) self-organize in chiral stacks in apolar solvents below a transition temperature. Here, the secondary interactions used for the supramolecular assembly are quadruple hydrogen bonds, $\pi-\pi$ stacking, and solvophobic effects.

Resonance energy transfer is fundamental to describe exciton dynamics in conjugated structures. It has been shown that intermolecular energy transfer is the dominant mechanism in polymer films while intramolecular energy transfer determines exciton dynamics in dilute polymer solutions. ${ }^{17-19}$ A quantitative study of intermolecular energy transfer in polymer films is complicated by the intrinsic positional and energy disorder of such systems. MOPV, on the other hand, is a model system to study energy transfer dynamics as it is possible to investigate the effect of intermolecular interactions by comparing optical properties of MOPV in the dissolved phase and the supramolecular assemblies. In supramolecular assemblies, the conjugated segments are closely packed in a fashion similar to conjugated segments in polymeric films but in a well-defined manner. In a previous paper, we have investigated exciton dynamics in MOPV at low excitation fluence with time-resolved photoluminescence spectroscopy and we found them to be very similar to those of polymeric films. ${ }^{20}$ Fast exciton diffusion on chiral supramolecular assemblies was shown to lead to exciton trapping and luminescence depolarization.

It has been documented that at sufficiently high excitation fluence, exciton dynamics are dominated by bimolecular annihilation processes. This exciton-exciton interaction has been studied in several polymeric systems ${ }^{21-30}$ (see Gadermaier and Lanzani ${ }^{31}$ for a recent review on ultrafast studies of exciton dynamics in polymeric semiconductors). Two 
models have been proposed for the mechanisms of excitonexciton annihilation and have been applied to various polymeric systems. The first model assumes the rate-limiting process to be the diffusion and encounter of excitons, with the rate constant being time independent. ${ }^{21,26,28-30}$ In the second model, the rate-limiting process is considered to be a longrange resonance energy transfer between the excitons. The proposed form of the rate constant varies but usually has an explicit time dependence due to the process being non-Markovian. ${ }^{22-25,27}$ These two mechanisms are in competition, and the key parameter that determines the importance of the non-Markovian mechanism is the spectral overlap of the $1^{1} B_{u}$ absorption and emission spectra. ${ }^{28}$ The excitedstate absorption spectrum is generally broad and its position is similar in polyphenylene and polyphenylenevinylene materials, but the PL spectral position is more sensitive to chemical composition. As a result, exciton bimolecular interactions in green- and red-emitting materials tend to be dominated by long-range resonance effects, while in blue-emitting materials collisional processes tend to dominate bimolecular annihilation dynamics.

In this paper we describe a comprehensive study of exciton bimolecular annihilation in MOPV supramolecular assemblies in dilute solution. Applying femtosecond transient absorption spectroscopy, we show that excitation with pulse fluences above $\sim 50 \mu \mathrm{J} / \mathrm{cm}^{2}$ results in bimolecular annihilation dynamics that dominate on picosecond time scales. This is in analogy to solid films of polymeric semiconductors. Long-range exciton bimolecular interactions determine the annihilation dynamics as has been reported for related alkoxy-substituted polyphenylenevinylene films. These results demonstrate that the electronic properties of solutionself-assembled supramolecular architectures are similar to those of disordered polymeric semiconductors. However, supramolecular electronics allow tailored control of intermolecular electronic interactions, as well as reversibility of the assembly process.

\section{EXPERIMENT}

The synthesis of MOPV4 (see Fig. 1 for the molecular structure) has been described elsewhere. ${ }^{15}$ The material was dissolved in anhydrous dodecane at a concentration of 4 $\times 10^{-4} \mathrm{M}$. Measurements were carried in a temperaturecontrolled absorption cuvette with $1 \mathrm{~mm}$ path length.

The femtosecond transient absorption apparatus consisted of a laser system based on the design of Backus et al. ${ }^{32}$ Briefly, 15-fs pulses were produced by a mode-locked Ti:sapphire oscillator (KMLabs TS pumped by $4.5 \mathrm{~W}, 532$ $\mathrm{nm}$ output from a Spectra-Physics Millennia V laser). The pulse train was amplified using a chirped-pulse-amplification scheme at a repetition rate of $1 \mathrm{kHz}$. The amplifier was a multipass Ti:sapphire amplifier (pumped by the $9.5 \mathrm{~W}, 527$ $\mathrm{nm}$ output from a Spectra-Physics Evolution X laser). The resulting pulses had a pulse width of $100 \mathrm{fs}$ and a pulse energy of $400 \mu \mathrm{J}$ and were centered at a wavelength of 800 $\mathrm{nm}(1.55 \mathrm{eV}$ photon energy).

The sample was excited with $3.1 \mathrm{eV}$ pump pulses produced by frequency doubling the laser fundamental in a 0.5 -

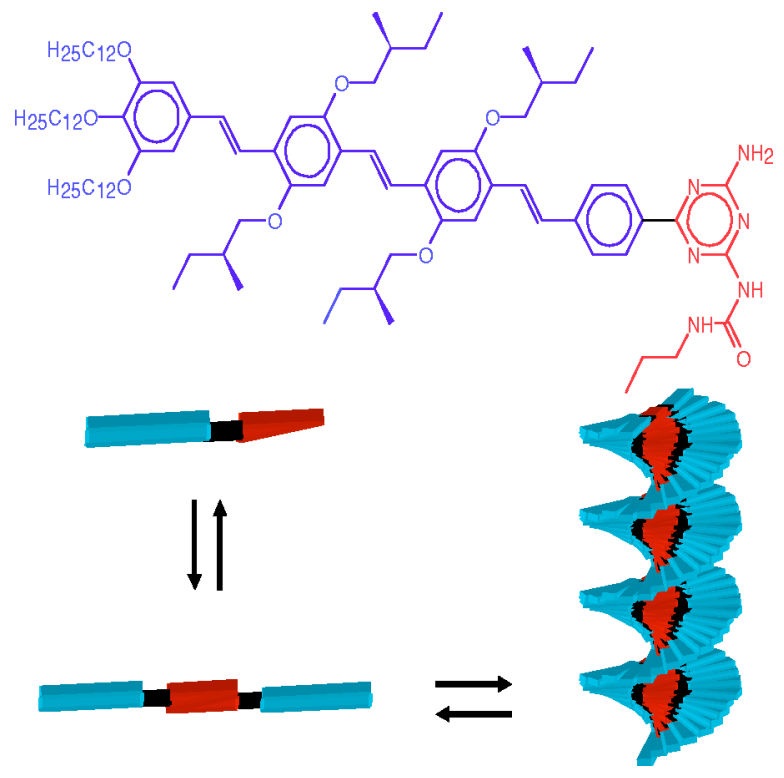

FIG. 1. (Color online) Molecular structure of MOPV4. In dodecane, quadrupolar hydrogen bonding of the ureido-s-triazine unit leads to the formation of dimers, which self-assemble into chiral structures as depicted in the schematic diagram. The supramolecular assembly is thermotropically reversible with a transition temperature of $75^{\circ} \mathrm{C}$ at an MOPV4 concentration of $4 \times 10^{-4} \mathrm{M}$.

mm-path-length $\beta$-BBO crystal. The pump power was varied with calibrated neutral density filters and the beam was mechanically chopped at $500 \mathrm{~Hz}$. The transient absorption of the sample was probed with a single-filament white-light continuum (approximately from $1.2 \mathrm{eV}$ to $3.0 \mathrm{eV}$ ) produced by focusing a fraction of the fundamental into a sapphire window. Probe pulses were delayed with respect to pump pulses with a computer-controlled optical delay. All the beams were linearly polarized with parallel polarization. The pump and the probe beams were focused on the sample cell to, respectively, $\sim 50 \mu \mathrm{m}$ and $100 \mu \mathrm{m}$ spots (with an angle between beams of approximatively $20^{\circ}$ ). The probe beam and a reference beam (not transmitted through the sample) were dispersed in a $0.25-\mathrm{m}$ spectrometer and detected with a pair of Si photodiodes. Pump-induced variation in transmission $(\Delta T / T)$ was extracted using standard lock-in techniques. The instrument response function had a full width at half maximum of less than $120 \mathrm{fs}$.

We applied time-correlated single-photon counting (TCSPC) to measure excited-state lifetimes. The MOPV solution was excited with a pulsed [20 MHz, 70 ps full width at half maximum (FWHM), $407 \mathrm{~nm}$ ] diode laser (PicoQuant LDH400). The luminescence was detected with a microchannel plate photomultiplier (Hamamatsu) coupled to a spectrometer and TCSPC electronics (Edinburgh Instruments Lifespec-ps and VTC900 PCI card). A decay curve was taken at $2.21 \mathrm{eV}$ photon energy, and the decay time was extracted from a monoexponential fit to the data.

\section{RESULTS AND ANALYSIS}

The material investigated here is an oligophenynevinylene consisting of four phenyl rings, monofunctionalized with a 


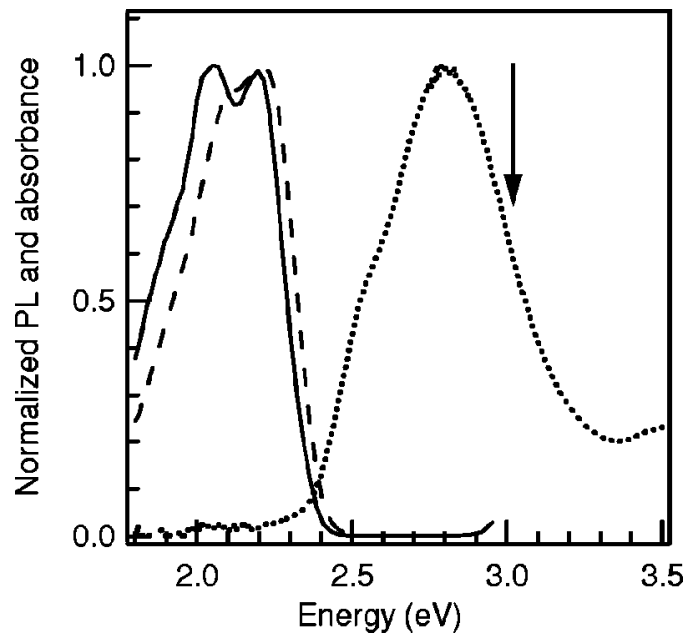

FIG. 2. Absorption spectrum of MOPV4 at $20^{\circ} \mathrm{C}$ (dotted line), PL spectrum at $14{ }^{\circ} \mathrm{C}$ (solid line), and PL spectrum at $65^{\circ} \mathrm{C}$ (dashed line). The black arrow indicates the excitation photon energy in all experiments reported here.

ureido-s-triazine (MOPV4) to dimerize by hydrogen bonding (see Fig. 1). The absorption spectrum of MOPV4 is shown in Fig. 2 together with the photoluminescence (PL) spectrum at $14{ }^{\circ} \mathrm{C}$ and $65^{\circ} \mathrm{C}$ for excitation using femtosecond pulses with photon energy just above the peak of the $\pi-\pi^{*}$ band. A redshift is observed upon cooling the solution, which has been previously attributed to the formation of supramolecular assemblies. ${ }^{15,20}$ Small-angle neutron scattering experiments showed that the average stack length is around $160 \mathrm{~nm}$ with a radius around $6 \mathrm{~nm} \cdot{ }^{33}$ The stack configuration is not known precisely, but according to preliminary quantumchemical calculations, the oligomer separation is approximatively $3.5 \AA$ and the angle of rotation between two neighboring oligomer is close to $12^{\circ} .{ }^{34}$ The intermolecular coupling in the stacks modifies the excitonic characteristics and leads to the appearance of a red shoulder in the PL spectrum. Above a certain transition temperature, which depends on the MOPV4 concentration, the thermodynamic equilibrium shifts from supramolecular assembly to a dissolved phase. ${ }^{15}$ Within a relatively narrow range $\left( \pm \sim 10{ }^{\circ} \mathrm{C}\right)$ about the transition temperature, the positional disorder in the stack increases and the average assembly length decreases. At the MOPV4 concentration used here, the transition temperature is around $\sim 75^{\circ} \mathrm{C}$. However, the two PL spectra show that at $65^{\circ} \mathrm{C}$, the red shoulder has already decreased significantly, suggesting that the intermolecular coupling is much smaller at $65{ }^{\circ} \mathrm{C}$. Therefore, we can use these two experimental conditions $\left(14\right.$ and $65^{\circ} \mathrm{C}$ ) to compare exciton dynamics with and without strong intermolecular coupling.

Figure 3 shows the excitation-fluence dependence of the time- and wavelength-integrated PL intensity of MOPV4 at $14{ }^{\circ} \mathrm{C}$ and $65^{\circ} \mathrm{C}$. The dependence is linear with excitation fluence but a sublinear trend appears at higher fluence. The transition between the two regimes is temperature dependent. At lower solution temperatures, the PL intensity saturates at significantly lower fluence. Moreover, the deviation from linearity at high excitation fluence is stronger at $14^{\circ} \mathrm{C}$. Such behavior is indicative of a time-integrated exciton population

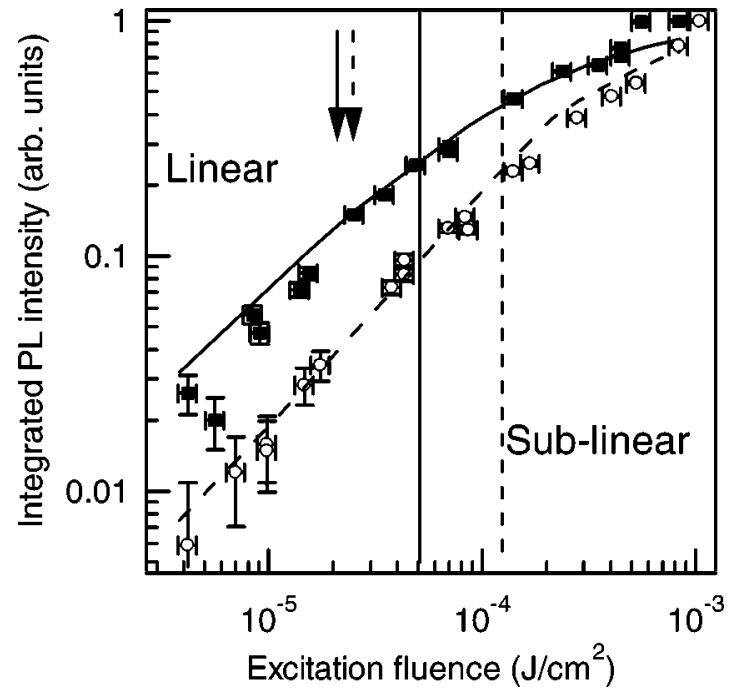

FIG. 3. Time- and wavelength-integrated PL intensity at $14{ }^{\circ} \mathrm{C}$ (solid squares) and at $65{ }^{\circ} \mathrm{C}$ (open circles). The solid and dashed lines are the results of fits to the time- and volume-integrated exciton population density using the model described in the text. The vertical lines separate linear and sub-linear regimes for the data taken at $14{ }^{\circ} \mathrm{C}$ (solid line) and $65^{\circ} \mathrm{C}$ (dashed line). The two arrows point to the excitation fluence of the data used for the stretched exponential fits (see Fig. 4).

which develops linearly with low pump fluence and sublinearly with high fluence. This indicates that exciton-exciton annihilation occurs on the supramolecular assemblies at high fluence. At low fluence, on the other hand, we are able to probe a regime where exciton-exciton annihilation dynamics are negligible.

Various OPV derivatives have been previously studied using femtosecond transient absorption spectroscopy, ${ }^{22,35-37}$ and three distinct spectral signatures were identified. The first is probe-induced stimulated emission (SE) signal overlapped with the PL spectrum (around $2.21 \mathrm{eV}$ ) while the other responses are photoinduced absorption (PA) signal around $1.77 \mathrm{eV}$ and $1.46 \mathrm{eV}$. The SE and 1.46-eV PA were assigned to $1{ }^{1} B_{u}$-exciton dynamics while the $1.77-\mathrm{eV}$ PA signal was assigned to polaron absorption. In the following discussion we will limit ourselves to the study of the $1^{1} B_{u}$-exciton dynamics, as the PA signal due to polaron absorption is strongly fluence dependent due to two-step exciton dissociation by resonant sequential excitation. ${ }^{30}$

Figure 4 shows the time evolution of a transient PA signal recorded at a probe photon energy of $1.46 \mathrm{eV}$ for low excitation fluence $\left(\sim 20 \mu \mathrm{J} \mathrm{cm}^{-2}\right)$. This corresponds to the regime where the PL intensity depends linearly on the excitation fluence (see the arrows in Fig. 3) and we can therefore neglect exciton-exciton interactions. The transient signal measured at $65{ }^{\circ} \mathrm{C}$ (open squares) decays quasiexponentially while the decay of the $14{ }^{\circ} \mathrm{C}$ signal (solid circles) displays a much faster initial component. The exponential component is assigned to the decay of localized excitons on isolated oligomers. We have shown previously that well below the transition temperature, the exciton dynamics is dominated by fast diffusion within the chiral supramolecular assembly, which 


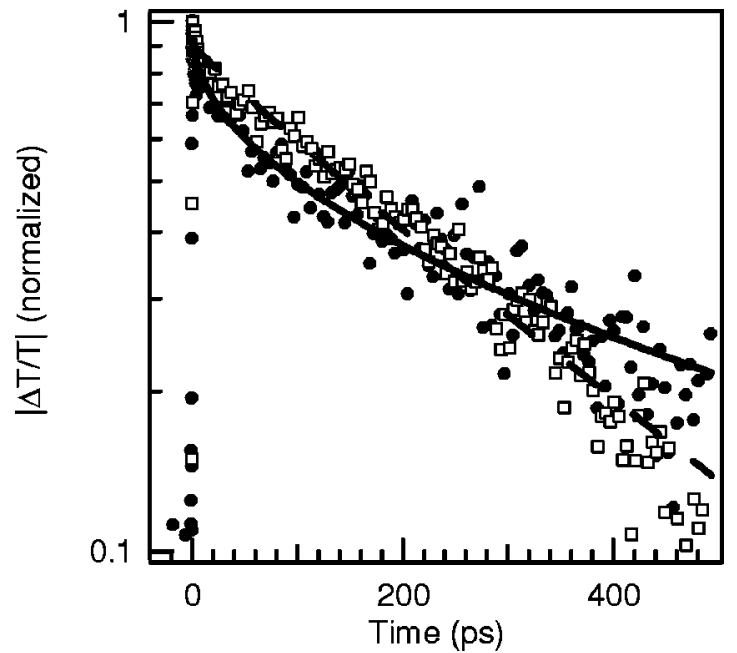

FIG. 4. Absorption transients at a probe photon energy of 1.46 $\mathrm{eV}$ and solution temperature of $14{ }^{\circ} \mathrm{C}\left(21 \mu \mathrm{J} \mathrm{cm}^{-2}\right.$ pump fluence, solid circles) and $65{ }^{\circ} \mathrm{C}\left(25 \mu \mathrm{J} \mathrm{cm}^{-2}\right.$ pump fluence, open squares) with fits to the expression $\left[c \exp (-t / \tau-t / a)^{b}\right]$. The solid line is fit to the data at $14{ }^{\circ} \mathrm{C}(a=300 \mathrm{ps}, b=0.4$, and $\tau=1600 \mathrm{ps})$ and the dashed line is the fit to the data at $65^{\circ} \mathrm{C}(a=300 \mathrm{ps}, b=0.9$, and $\tau=1600 \mathrm{ps}$ ).

leads to trapping and subsequent slower transfer of localized excitons. ${ }^{20}$ The ultrafast decay of the signal measured at $14{ }^{\circ} \mathrm{C}$ can therefore be interpreted as a consequence of exciton diffusion along the stacks.

We have measured time-dependent SE and PA transient signals in MOPV4 solution at various pump fluences, probing at $2.21 \mathrm{eV}$ (Fig. 5) and $1.46 \mathrm{eV}$ (Fig. 6), respectively. The dynamics of both signals measured at $14{ }^{\circ} \mathrm{C}$ depend on the excitation fluence, and at higher fluence are considerably faster than those measured at $65^{\circ} \mathrm{C}$. The fast decay present within the first 10 ps at $14{ }^{\circ} \mathrm{C}$ increases in relative contribution and becomes faster as the excitation fluence is increased. At those fluences, the initial PA and SE signals (at $t=0$ ) have a sublinear dependence in the excitation fluence (Fig. 7). The deviation from linearity is stronger in the data measured at $14{ }^{\circ} \mathrm{C}$ than for those measured at $65^{\circ} \mathrm{C}$. Also, the transition between linear and sublinear regimes at $14{ }^{\circ} \mathrm{C}$ occurs at lower fluence. These results are consistent with the fluence dependence of the integrated PL intensity (Fig. 3).

The sublinear excitation fluence dependences displayed in Figs. 3 and 7 are characteristic of bimolecular exciton annihilation dynamics. Since the transient absorption signal decay rates depend on the excitation fluence at $14{ }^{\circ} \mathrm{C}$, we correlate the exciton-exciton annihilation processes with the formation of supramolecular assemblies. At $14{ }^{\circ} \mathrm{C}$, exciton diffusion on the supramolecular stacks is fast enough to allow for exciton-exciton interactions at sufficiently high density. At $65{ }^{\circ} \mathrm{C}$, localization of excitons becomes dominant as the disorder is increased. ${ }^{20}$ Furthermore, just below the transition temperature, the average stack length is shorter as the equilibrium shifts towards the dissolved phase ${ }^{33}$ so that the exciton density required for exciton-exciton interaction is higher.

In order to obtain a more quantitative description of exci-

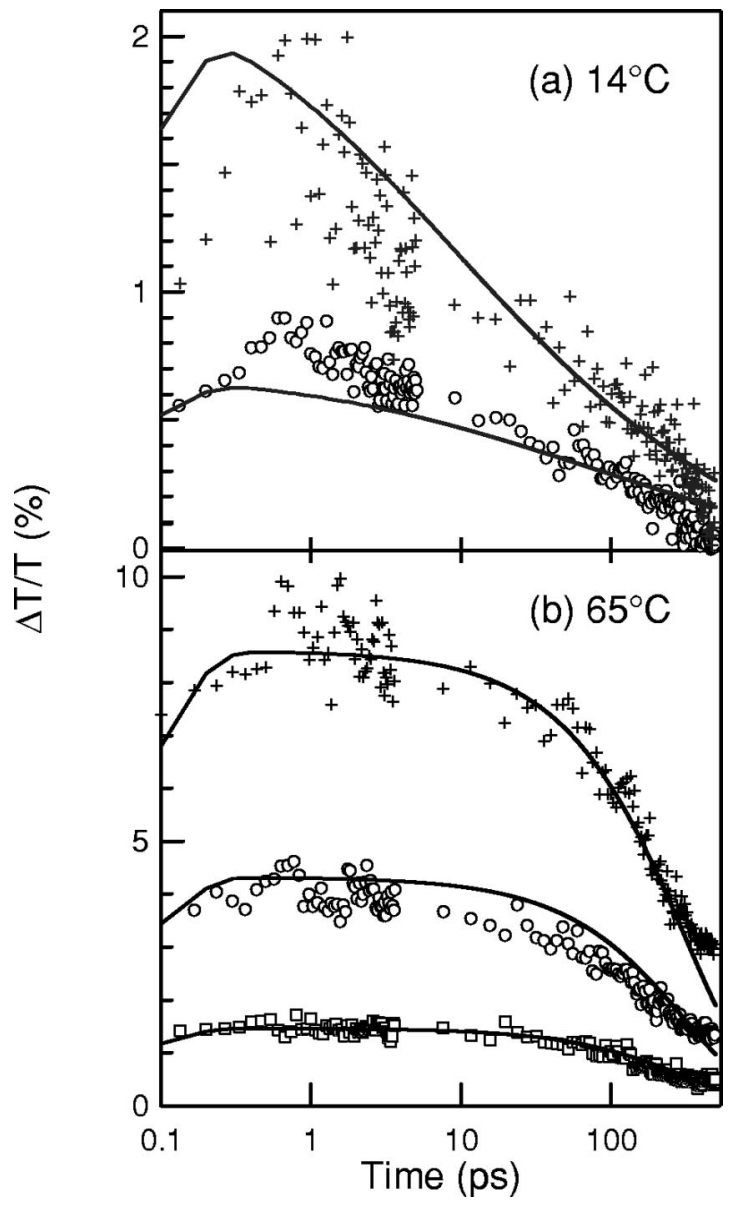

FIG. 5. (a) SE signal (probe energy $2.21 \mathrm{eV}$ ) at $14{ }^{\circ} \mathrm{C}$ for $279 \mu \mathrm{J} \mathrm{cm}^{-2}$ (crosses) and $111 \mu \mathrm{J} \mathrm{cm}^{-2}$ (circles) pump fluence. The lines are fits with the photophysical model at the corresponding pump fluence. (b) SE signal (probe energy $2.21 \mathrm{eV}$ ) at $65{ }^{\circ} \mathrm{C}$ at $836 \mu \mathrm{J}, \mathrm{cm}^{-2}$ (crosses), $209 \mu \mathrm{J} \mathrm{cm}^{-2}$ (circles), and $70 \mu \mathrm{J} \mathrm{cm}^{-2}$ (squares) pump fluence. The lines are fits to the model at the corresponding pump fluence.

ton bimolecular annihilation dynamics in MOPV4 nanostructures, we have constructed a mesoscopic model that reproduces our experimental results and allows us to unravel the mechanism governing the annihilation process. The model is based on a rate equation for the exciton population $N$, given by

$$
\frac{d N}{d t}=G(t)-\frac{N}{\tau}-\beta N^{2}-\gamma \frac{N^{2}}{t^{d}}-\epsilon N\left(\frac{t}{a}\right)^{b-1} .
$$

The first term is the exciton generation rate, which has the form

$$
G(t)=\frac{\alpha P \lambda}{2 \pi r^{2} h c} \frac{N_{g r}-S(t)}{N_{g r}} \frac{\exp \left[-\left(t-t_{0}\right)^{2} / 2 \sigma_{t}^{2}\right]}{\sqrt{2 \pi \sigma_{t}^{2}}},
$$

where $P$ is the excitation pulse energy, $\alpha$ is the unsaturated absorbtion coefficient, $\lambda$ is the excitation wavelength, $r$ is the Gaussian radius of the excitation beam, $S(t)$ is the number of excitons created between $t_{0}$ and $t$ (with $t_{0}$ the zero time), $N_{g r}$ 


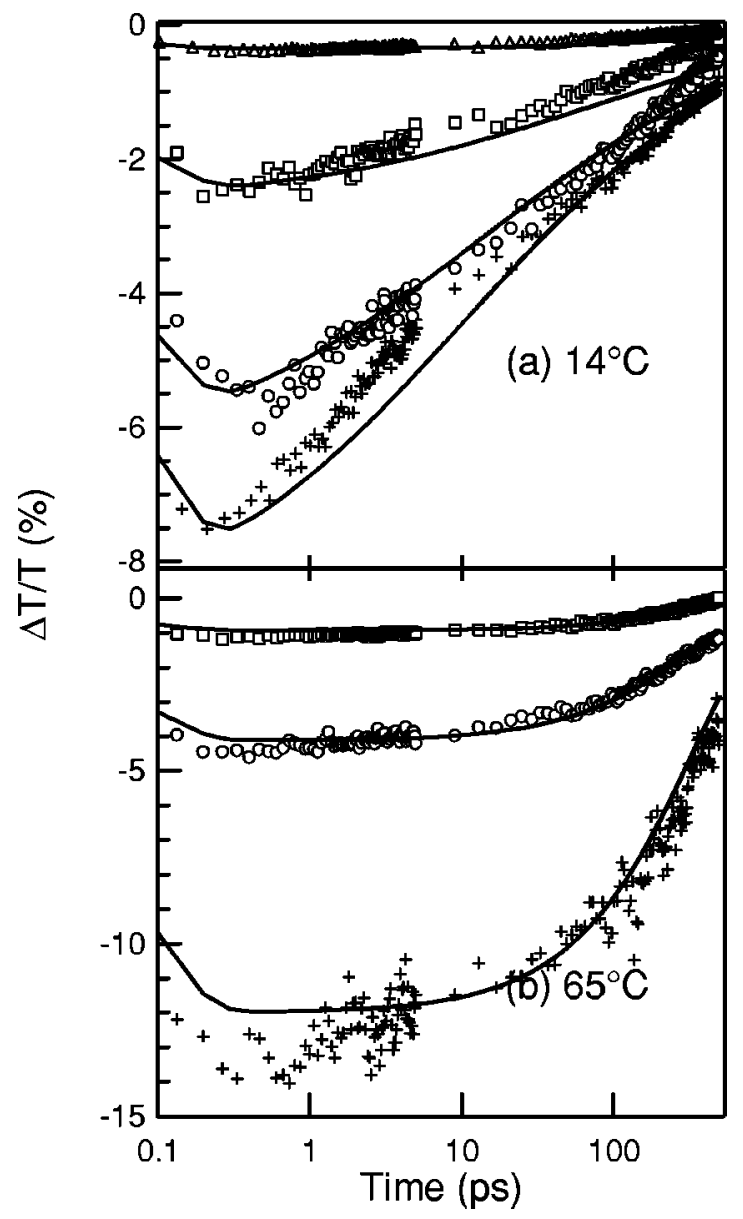

FIG. 6. (a) PA signals (probe energy $1.46 \mathrm{eV}$ ) at $14{ }^{\circ} \mathrm{C}$ for pump fluence of $557 \mu \mathrm{J} \mathrm{cm}^{-2}$ (crosses), $209 \mu \mathrm{J} \mathrm{cm}^{-2}$ (circles), $84 \mu \mathrm{J} \mathrm{cm}^{-2}$ (squares), and $21 \mu \mathrm{J} \mathrm{cm}^{-2}$ (triangles). (b) PA signals (probe energy $1.46 \mathrm{eV}$ ) at $65{ }^{\circ} \mathrm{C}$ for pump fluence of $557 \mu \mathrm{J} \mathrm{cm}^{-2}$ (crosses), $111 \mu \mathrm{J} \mathrm{cm}^{-2}$ (circles), and $25 \mu \mathrm{J} \mathrm{cm}^{-2}$ (squares). The lines are the fits to the data at corresponding pump fluence according to the model described in the text.

is the initial density of ground states, and $\sigma_{t}$ is the width of the excitation pulse. To calculate $S(t)$, we use

$$
S(t+d t)=G(t) d t .
$$

The generation term takes into account the Gaussian distribution in space and time of the excitation pulse. The initial density of ground states is taken to be the concentration of MOPV4 molecules with the ground-state depletion taken into account in each point.

The second term in Eq. (1) describes the unimolecular excited-state decay rate, with time constant $\tau$ measured using TCSPC for an MOPV4 solution at 14 and $65{ }^{\circ} \mathrm{C}$ at a very low excitation fluence. Collisional bimolecular annihilation processes are described by the third term, where $\beta$ is the diffusion-limited exciton bimolecular annihilation rate constant. The general form includes a time dependence but this term may be neglected if the typical diffusion length on experimental time scales is larger than the extent of the exciton. ${ }^{25,38}$ In conjugated systems, this is usually the case; however, this assumption will be discussed further in Sec. IV.

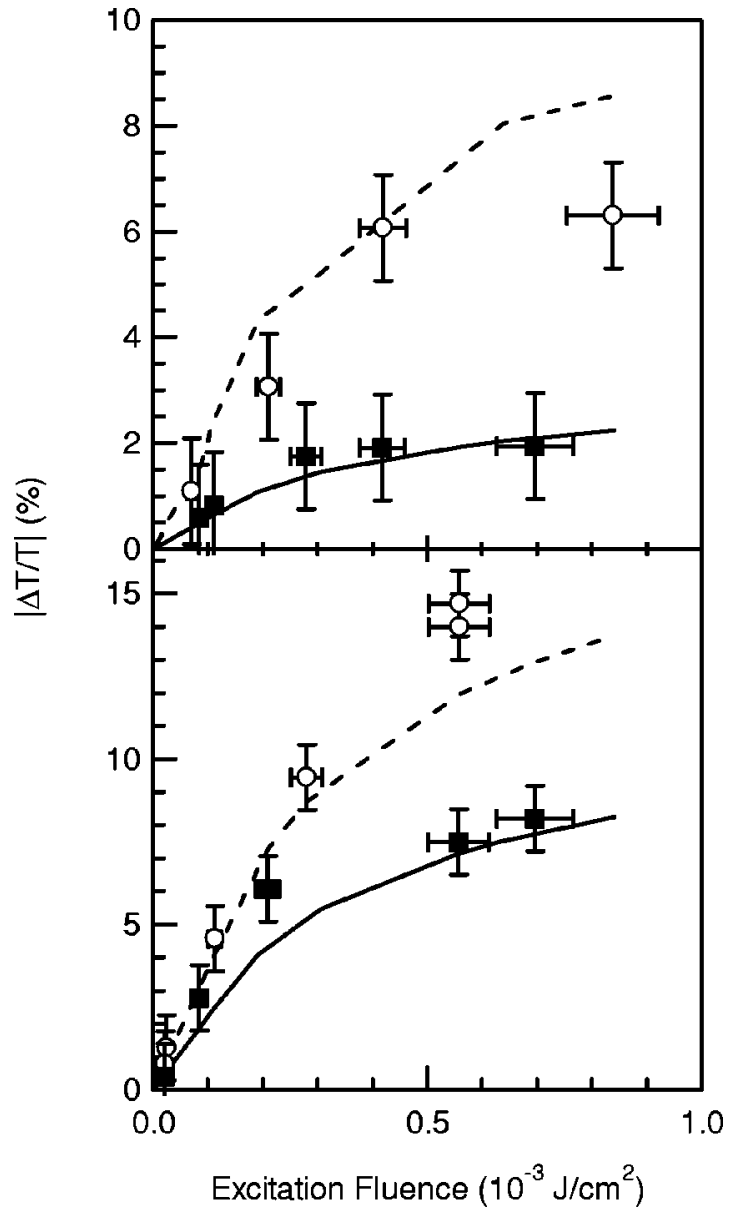

FIG. 7. (a) SE signal (probe energy $2.21 \mathrm{eV}$ ) at $t=0$ as a function of excitation fluence at $14{ }^{\circ} \mathrm{C}$ (solid squares) and at $65{ }^{\circ} \mathrm{C}$ (open circles). This amplitude was determined by fitting the transient absorption data in Figs. 5 and 6 with a convolution of the instrument response function and a multiexponential function and extrapolating to zero time. This procedure allows accurate deconvolution of the instrument response. (b) PA signals (probe energy $1.46 \mathrm{eV}$ ) at $t=0$ as a function of excitation fluence at $14{ }^{\circ} \mathrm{C}$ (solid squares) and at $65^{\circ} \mathrm{C}$ (open circles), determined as described above. The lines through all data are fits to the photophysical model described in the text.

On the other hand, the long-range time-dependent bimolecular annihilation term, with rate constant $\gamma$, includes explicit time dependence. This time dependence was introduced following resonance energy transfer studies and accounts for the dispersive nature of the transfer process resulting from the distribution of transfer rates in a random ensemble of donors and acceptors, which leads to a nonMarkovian depletion of acceptors near the donors. The exact time dependence, characterized by the time exponent $d$, varies with the spatial distribution of acceptors and depends on dimensionality. ${ }^{39,40}$ However, other factors such as disorder may also lead to a power-law dependence of the transfer rate (this point will be discussed further in Sec. IV) and we have therefore considered $d$ in this term as a fitting variable.

The final term in Eq. (1) represents the ultrafast dynamics related to exciton diffusion in supramolecular assemblies. We have reported previously dispersive diffusion dynamics of 
excitons in MOPV4 and we will therefore not consider these issues in detail here. ${ }^{20}$ To take into account effects of diffusion to nonradiative traps, we have added a term in our rate equation so that at low excitation fluence the solution converges towards the low-excitation-fluence data. To determine this term, we fit the PA signal measured at the lowest excitation fluence (within the linear intensity regime; see Fig. 4) with a stretched exponential, taking into account the natural decay $\left(\exp \left[-t / \tau-(t / a)^{b}\right]\right)$, where $a$ is the stretchedexponential time constant and $b$ is the exponent in the stretched exponential. In Eq. (1), $\epsilon$ is the amplitude of the contribution of this dispersive process. This method follows theoretical $^{41-43}$ and experimental ${ }^{44,45}$ studies of exciton diffusion to traps were a stretched-exponential behavior was obtained. The stretched-exponential fit to the $65{ }^{\circ} \mathrm{C}$ PA data is very close to an exponential function but the time dependence of the PA data at $14{ }^{\circ} \mathrm{C}$ is close to an exponential of the square root of time. This indicates that diffusion to traps and polarization decay influence the PA signal dynamics at $14{ }^{\circ} \mathrm{C}$ but are less important at $65^{\circ} \mathrm{C}$, as established by our previous studies of exciton dynamics on MOPV4 at low fluence. $^{20}$

The experimental geometry is taken into account in the photophysical model by dividing the volume located at the intersection of the probe and pump beams into small subvolumes with homogeneous excitation fluence where the rate equation can be solved. The measured quantities are then calculated by summing the exciton densities in a mesoscopic approach. To calculate the SE and PA signals, the fraction of probe photons transmitted through the sample needs to be evaluated. This fraction is related to the exciton density and to the SE and PA cross sections by

$$
d \rho_{\nu}= \pm \sigma N \rho_{\nu} d x
$$

where $\rho_{\nu}$ is the photon density, $\sigma$ is the cross section for SE or for PA, and $x$ is the sample depth. As we are only interested in the sample transmission, the initial probe photon population is arbitrary. The average exciton density at a particular sample depth is determined in a similar manner as the total exciton density on the entire volume but the multiplication factors needed are the surface fractions excited with each of the particular excitation fluence of the set, at the particular sample depth.

The parameter $\tau$ is obtained separately from TCSPC measurements, $N_{g r}$ from the knowledge of the MOPV4 concentration, $\alpha$ and therefore $\sigma$ (defined as $\alpha / N_{g r}$ ) from UV-vis absorption measurements, and $a$ and $b$ from the stretchedexponential fitting of the low fluence PA signal. To determine the remaining parameters $\left(\beta, \gamma\right.$, and $d ; \epsilon, \sigma_{P A}$, and $\left.\sigma_{S E}\right)$, the PA and SE signals at all excitation fluence, the zero-time signals, and the integrated PL data are modeled using a global fit. The best results of the fitting procedure are displayed in Figs. 3, 5, 6, and 7 with the model parameters summarized in Table I.

A high bimolecular annihilation rate constant $\gamma$ was needed to fit the time-resolved data taken at $14{ }^{\circ} \mathrm{C}$, but bimolecular annihilation effects were not necessary to reproduce the data taken at $65{ }^{\circ} \mathrm{C}$. We find that the magnitude of
TABLE I. Summary of parameters used in the photophysical model.

\begin{tabular}{lcc}
\hline \hline & $14{ }^{\circ} \mathrm{C}$ & $65^{\circ} \mathrm{C}$ \\
\hline$\tau(\mathrm{ps})$ & 2940 & 2940 \\
$\beta\left(\mathrm{cm}^{3} \mathrm{ps}^{-1}\right)$ & 0 & 0 \\
$\gamma\left(\mathrm{cm}^{3} \mathrm{ps}^{d-1}\right)$ & $3.6 \times 10^{-17}$ & 0 \\
$d$ & 0.5 & - \\
$\epsilon\left(\mathrm{ps}^{-1}\right)$ & $2.2 \times 10^{-4}$ & $2.5 \times 10^{-3}$ \\
$a(\mathrm{ps})$ & 266 & 300 \\
$b$ & 0.46 & 0.9 \\
$\sigma\left(\mathrm{cm}^{2}\right)$ & $9.25 \times 10^{-17}$ & $9.25 \times 10^{-17}$ \\
$N_{g r}\left(\mathrm{~cm}^{-3}\right)$ & $2.49 \times 10^{17}$ & $2.49 \times 10^{17}$ \\
$\sigma_{P A}\left(\mathrm{~cm}^{2}\right)$ & $1.55 \times 10^{-16}$ & $2.5 \times 10^{-16}$ \\
$\sigma_{S E}\left(\mathrm{~cm}^{2}\right)$ & $4 \times 10^{-17}$ & $1.4 \times 10^{-16}$
\end{tabular}

$\gamma$ is almost an order of magnitude higher than the typical values obtained in polymer films. ${ }^{23-25,46}$ Furthermore, the data at $14{ }^{\circ} \mathrm{C}$ cannot be fitted with a time-independent annihilation term, and setting $\beta=0$ did not compromise the global fits presented here. Variation of the exponent $d$ between 0.4 and 0.7 does not have a significant effect on the quality of the fit; however, the best fit is obtained with a square-root dependence in time $(d=0.5)$. Figure 8 shows the exciton density at the maximum experimental fluence used here $\left(\sim 840 \mu \mathrm{J} \mathrm{cm}^{-2}\right)$, as determined by the model. The timedependent bimolecular annihilation term leads to an ultrafast decay of the exciton density at $14{ }^{\circ} \mathrm{C}$, but is insignificant at $65^{\circ} \mathrm{C}$. Note that the peak exciton population density at both temperatures $\left(<5 \times 10^{15} \mathrm{~cm}^{-3}\right)$ is $1.8 \%$ of the ground-state chromophore density used in these experiments $\left(N_{g r}\right.$ in Table I), and therefore photophysical saturation is not the origin of sublinear fluence dependence observed in Figs. 3 and 7. Such low exciton densities in the supramolecular nanostructure point to the need for a high bimolecular annihilation rate constant at the photon flux regime used here.

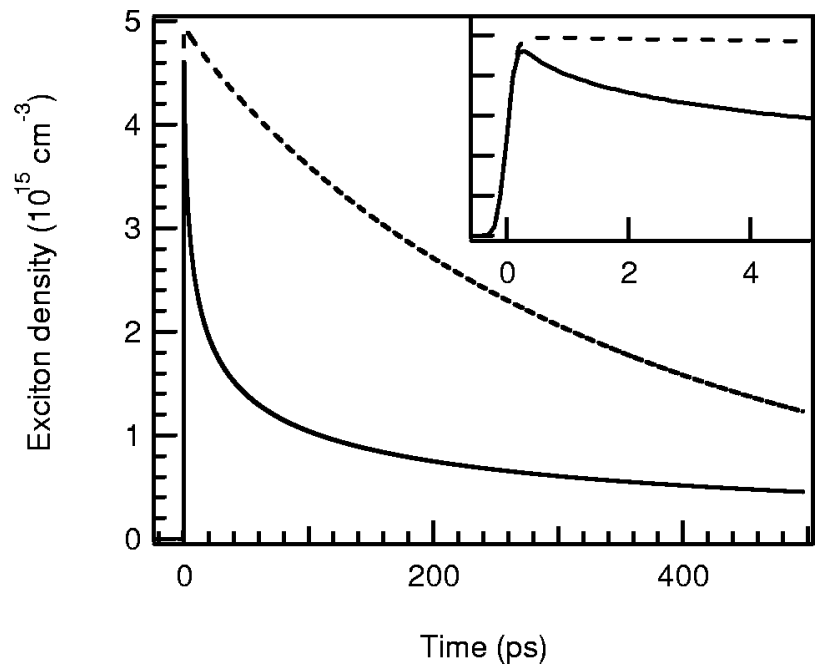

FIG. 8. Exciton density at peak excitation fluence against time for the parameters reported in Table I at $14{ }^{\circ} \mathrm{C}$ (solid line) and $65^{\circ} \mathrm{C}$ (dashed line). Inset: data plotted over the first 5 ps. 


\section{DISCUSSION}

Our model shows that exciton bimolecular annihilation is necessary to describe excited-state dynamics in MOPV4 solution at $14{ }^{\circ} \mathrm{C}$ while it has a negligible effect at $65^{\circ} \mathrm{C}$. To check that the high value we obtain for the bimolecular annihilation constant is not a consequence of the mesoscopic character of our model, we performed a similar analysis to those developed in a range of previous studies. ${ }^{23,24,29}$ In this crude analysis, the exciton density was modeled with a simplified rate equation with a unimolecular decay term, a timedependent bimolecular annihilation term, and a $\delta$ function as the generation term:

$$
\begin{gathered}
\frac{d N}{d t}=N_{0} \delta(t)-\frac{N}{\tau}-\gamma \frac{N^{2}}{\sqrt{t}}, \\
N=\frac{\exp (-t / \tau)}{N_{0}^{-1}+2 \gamma \sqrt{\tau} \int_{0}^{\sqrt{t / \tau}} \exp \left(-x^{2}\right) d x} .
\end{gathered}
$$

For small signals, the PA signal is directly related to the exciton population $\left(\Delta T / T \approx-\sigma_{P A} N x\right)$, so we also fitted our data with the analytical solution of the simplified rate equation. The exciton population at zero time, $N_{0}$, was approximated with the known density of absorbed photons, which allowed us to relate the fitting coefficient to the parameter $\gamma$. The value obtained from the high-fluence PA signals, $\gamma \approx 2$ $\times 10^{-17} \mathrm{~cm}^{3} \mathrm{ps}^{-1 / 2}$, is very similar to the result of our model, which is reproduced by this crude analysis but is closer to the MOPV4 physics as it takes into account the variation in excitation fluence in the sample.

Several studies have interpreted the square-root time dependence of the bimolecular annihilation as an effect of the non-Markovian depletion of close acceptor-donor pairs. With a point-dipole approximation, the Förster model predicts a time dependence of the excitation transfer rate of $t^{-(1-D / 6)}$, with $D$ being the dimensionality of the acceptor distribution. This result was obtained by applying the method developed by Eisenthal and Siegel for the three-dimensional case to the one- and two-dimensional cases. ${ }^{40}$ If this model is valid to describe the exciton-exciton annihilation, the dimensionality characteristic of exciton distribution in MOPV4 stacks is 3 (which corresponds to the best fit, with $d=0.5$, but a fractal dimensionality between 3 and 2 cannot be ruled out). The point-dipole model also relates the magnitude of the excitonexciton annihilation rate constant to the Förster radius by

$$
\begin{gathered}
\gamma^{D}=\frac{R_{0}^{D} \pi^{D / 2} \Gamma(2-D / 6)}{\Gamma(1+D / 2) \xi^{D / 6}}, \\
\gamma^{3}=\frac{2 \pi}{3} R_{0}^{3} \sqrt{\frac{\pi}{\xi}}
\end{gathered}
$$

where $\Gamma$ is the gamma function, $\xi$ the exciton lifetime in the absence of transfer, and $R_{0}$ the Förster radius. With $D=3$, we extract $R_{0} \approx 70 \mathrm{~nm}$ with our value of $\gamma$, which is unrealistic. However, the simple model is based on a random dis- tribution of acceptors in a sphere of dimensionality $D$ centered on the donor and it fails to reproduce a distribution of excitons in the complex geometry of MOPV4 stacks. We also point out the limitations of the applicability of Förster theory in organic semiconductor systems, where the pointdipole approximation is often unjustified. ${ }^{18}$

The collisional mechanism used in the model does not include any time dependence. This is justified if the typical diffusion length on experimental time scales is larger than the spatial extent of the exciton. However, the general expression in three dimensions includes a time dependence given by ${ }^{38}$

$$
\beta=8 \pi R_{e} D\left(1+\frac{R_{e}}{\sqrt{\pi D t}}\right),
$$

with $D$ the diffusion coefficient and $R_{e}$ the collision distance. If we assign the observed square-root time dependence to this simple collisional mechanism, we obtain an unrealistic value for the diffusion coefficient, $D \approx 10^{5} \mathrm{~cm}^{2} \mathrm{~s}^{-1}$ with $R_{e}=1 \mathrm{~nm}$. The collision radius $R_{e}$ would have to be around $50 \mathrm{~nm}$ for $D$ to be similar to the values found in organic systems $\left(D \approx 10^{-2} \mathrm{~cm}^{2} \mathrm{~s}^{-1}\right) .{ }^{38}$ Furthermore, such high values of $D$ correspond to the time-independent regime so that the collisional mechanism cannot explain our data. If we use the value of $\beta$ corresponding to the best global fit (which is significantly worse than that shown in Figs. 3, 5, 6, and 7), realistic values for the diffusion coefficient can be obtained if $R_{e} \geqslant 5 \mathrm{~nm}$. ${ }^{38}$ The expression used here corresponds to diffusion in a three-dimensional lattice, but similar expressions exist for the one- and two-dimensional cases. ${ }^{47}$ All of these fail to reproduce the observed bimolecular exciton annihilation time dependence and intensity dependence.

With the above considerations, we can conclude that neither the collisional model nor the Förster (point-dipole) model can uniquely explain the high rate of exciton bimolecular annihilation measured in MOPV4 stacks. These two mechanisms represent limiting cases where either incoherent exciton hopping or Förster energy transfer are sufficient to describe the annihilation. A more realistic description is an intermediate case of fast incoherent hopping followed by efficient resonance energy transfer. The square-root time dependence of the bimolecular annihilation term extracted from the model in Eq. (1) is an effect of the complex geometry of the supramolecular assemblies and of disorder. Combined with rapid exciton diffusion within the stack, ${ }^{20}$ bimolecular interactions lead to the high annihilation rate. A branching between exciton localization and direct bimolecular annihilation is likely at early time. At later times, bimolecular annihilation of localized excitons is likely. This picture is analogous to related studies of resonance energy transfer dynamics in blends containing small concentrations of MOPV4 in similar oligomers with shorter conjugation length. ${ }^{48}$

A more accurate description of the bimolecular annihilation dynamics would require comparing the time-dependent average exciton density accessed with our measurement and a configurationally averaged microscopic description of the transfer processes using a Monte Carlo or a master equation approach. Implementation of these schemes requires a sig- 
nificant number of assumptions, including detailed understanding of the structural and geometrical disorder. This information is beyond the scope of the present study.

On the other hand, a detailed description should be based on a quantum-chemical, non-Markovian model where the exciton-exciton coupling and electron-phonon coupling are explicitly taken into account. OPV systems have been shown to belong to the intermediate-coupling regime where the relaxation energy accompanying optical excitation, the nearestneighbor exciton-exciton coupling, and the frequency of the most strongly coupled intramolecular vibration are comparable. ${ }^{49}$ In MOPV4 supramolecular stacks, the intermolecular exciton-exciton coupling is comparable to the typical intramolecular exciton-exciton coupling found in polymeric systems. ${ }^{34}$ For such an intermediate case between polymeric systems and molecular aggregates that undergo coherent excitation transfer (supertransfer), the description of exciton-exciton interactions would need to go beyond a Förster description of energy transfer. ${ }^{50,51}$

We are currently exploring both microscopic semiclassical and quantum-chemical approaches. However, the work presented here demonstrates unambiguously that the exciton dynamics in supramolecular nanostructures in solution is similar to that observed in polymeric semiconductors, but control of intermolecular electronic interactions is higher such that high exciton bimolecular annihilation rates are measured.

\section{CONCLUSION}

We have shown that the exciton dynamics in supramolecular assemblies of MOPV4 is very similar to the dynamics observed in conjugated polymer films. Supramolecular chemistry is a very promising route to construct well-defined semiconductor architectures with polymeric properties through controlled reversible assembly.

We have applied femtosecond transient absorption spectroscopy as a means to explore exciton dynamics in a supramolecular nanostructure constructed with a hydrogen- bonded oligophenylenevinylene derivative. A simple rateequation model was applied successfully to separate exciton bimolecular annihilation phenomena from unimolecular processes occurring at low fluence. ${ }^{20}$ We have shown that exciton-exciton annihilation does take place on the supramolecular assemblies and dominates the exciton dynamics at high fluence. The mechanism for bimolecular annihilation is non-Markovian; explicit time dependence is necessary to reproduce the experimental data with the model. The rate constant for bimolecular annihilation is higher than in comparable organic systems and cannot be rationalized with the simple models used in previous studies. We consider that the electronic properties of this system fall in the intermediateelectronic-coupling regime, where intermolecular electronic coupling is comparable to the intramolecular reorganization energy, providing an opportunity to apply semiclassical and quantum-chemical methodologies to explore electronic dynamics in this promising regime of supramolecular electronics. We are currently pursuing this opportunity.

\section{ACKNOWLEDGMENTS}

The authors thank Professor Gregory Scholes, Dr. Stefan Meskers, and Dr. David Beljonne for fruitful discussions. The work in Cambridge is supported by the UK Engineering and Physical Sciences Research Council (EPSRC) and the interdisciplinary research center for nanotechnology (Cambridge, UCL, Bristol). C.S. acknowledges additional financial support from the EPSRC through an Advanced Research Fellowship. C.D. was partially supported by the Isaac Newton Trust and by the Cambridge Interdisciplinary Research Centre for Nanotechnology. L.M.H. thanks St. John's College, Cambridge for financial support. The work in Eindhoven is supported by the Netherlands Organization for Scientific Research (NWO, CW). A.P.H.J.S. is supported by the Royal Netherlands Academy of Arts and Science. The Cambridge-Eindhoven Collaboration is supported by the European Commission (LAMINATE).
*Corresponding author. Electronic address: cs271@cam.ac.uk; http://www-oe.phy.cam.ac.uk/fast/index.htm

${ }^{1}$ N. Stutzmann, R. H. Friend, and H. Sirringhaus, Science 299, 1881 (2003).

${ }^{2}$ C. D. Dimitrakopoulos and P. R. L. Malenfant, Adv. Mater. Sci. 14, 99 (2002).

${ }^{3}$ R. H. Friend et al., Nature (London) 397, 121 (1999).

${ }^{4}$ S. Forrest, P. Burrows, and M. Thompson, IEEE Spectrum 37, 29 (2000)

${ }^{5}$ C. J. Brabec, C. Winder, N. S. Sariciftci, J. C. Hummelen, A. Dhanabalan, P. A. van Hal, and R. A. J. Janssen, Adv. Funct. Mater. 12, 709 (2002).

${ }^{6}$ B. A. Gregg, J. Phys. Chem. 107, 4688 (2003).

${ }^{7}$ P. Peumans, A. Yakimov, and S. R. Forrest, J. Appl. Phys. 93, 3693 (2003).

${ }^{8}$ S. Setayesh, A. C. Grimsdale, T. Weil, V. Enkelmann, K. Müllen, F. Meghdadi, E. J. W. List, and G. Leising, J. Am. Chem. Soc. 123, 946 (2001).
${ }^{9}$ C. Ego, D. Marsitzky, S. Becker, J. Y. Zhang, A. C. Grimsdale, K. Müllen, J. D. Mackenzie, C. Silva, and R. H. Friend, J. Am. Chem. Soc. 125, 437 (2003).

${ }^{10}$ A. C. Grimsdale, P. Leclère, J. D. Mackenzie, C. Murphy, S. Setayesh, C. Silva, R. H. Friend, and K. Müllen, Adv. Funct. Mater. 12, 729 (2002).

${ }^{11}$ H. Sirringhaus, T. Kawase, R. H. Friend, T. Shimoda, M. Inbasekaran, W. Wu, and E. P. Woo, Science 290, 2123 (2000).

${ }^{12}$ T. Kawase, H. Sirringhaus, R. H. Friend, and T. Shimoda, Adv. Mater. (Weinheim, Ger.) 13, 1601 (2001).

13 J.-M. Lehn, Supramolecular Chemistry (VCH, Weinheim, Germany, 1995).

${ }^{14}$ A. M. van de Craats, J. M. Warman, A. Fechtenkötter, J. D. Brand, M. A. Harbison, and K. Müllen, Adv. Mater. (Weinheim, Ger.) 11, 1469 (1999).

${ }^{15}$ A. P. H. J. Schenning, P. Jonkheijm, E. Peeters, and E. W. Meijer, J. Am. Chem. Soc. 123, 409 (2001).

${ }^{16}$ E. W. Meijer and A. P. H. J. Schenning, Nature (London) 419, 353 (2002). 
${ }^{17}$ T.-Q. Nguyen, J. Wu, V. Doan, B. J. Schwartz, and S. H. Tolbert, Science 288, 652 (2000).

${ }^{18}$ D. Beljonne, G. Pourtois, C. Silva, E. Hennebicq, L. M. Herz, R. H. Friend, G. D. Scholes, S. Setayesh, K. Müllen, and J. L. Brédas, Proc. Natl. Acad. Sci. U.S.A. 99, 10982 (2002).

${ }^{19}$ C. Silva, D. M. Russell, A. S. Dhoot, L. M. Herz, C. Daniel, N. C. Greeham, A. C. Arias, S. Setayesh, K. Müllen, and R. H. Friend, J. Phys.: Condens. Matter 64, 125211 (2002).

${ }^{20}$ L. M. Herz, C. Daniel, C. Silva, F. J. M. Hoeben, A. P. H. J. Schenning, E. W. Meijer, R. H. Friend, and R. T. Phillips, Phys. Rev. B 68, 045203 (2003).

${ }^{21}$ R. G. Kepler, V. S. Valencia, S. J. Jacobs, and J. J. McNamara, Synth. Met. 78, 227 (1996).

${ }^{22}$ V. I. Klimov, D. W. McBranch, N. N. Barashkov, and J. P. Ferraris, Chem. Phys. Lett. 277, 109 (1997).

${ }^{23}$ D. Vacar, E. S. Maniloff, D. W. McBranch, and A. J. Heeger, Phys. Rev. B 56, 4573 (1997).

${ }^{24}$ E. S. Maniloff, V. I. Klimov, and D. W. McBranch, Phys. Rev. B 56, 1876 (1997).

${ }^{25}$ A. Dogariu, D. Vacar, and A. J. Heeger, Phys. Rev. B 58, 10218 (1998)

${ }^{26}$ G. J. Denton, N. Tessler, M. A. Stevens, and R. H. Friend, Synth. Met. 102, 1008 (1999).

${ }^{27}$ T. Q. Nguyen, I. B. Martini, J. Liu, and B. J. Schwartz, J. Phys. Chem. B 104, 237 (2000).

${ }^{28}$ M. A. Stevens, C. Silva, D. M. Russell, and R. H. Friend, Phys. Rev. B 63, 165213 (2001).

${ }^{29}$ M. Shimizu, S. Suto, A. Yamamoto, and T. Goto, Phys. Rev. B 64, 115417 (2001).

${ }^{30}$ C. Silva, A. S. Dhoot, D. M. Russell, M. A. Stevens, A. C. Arias, J. D. MacKenzie, N. C. Greenham, S. Setayesh, K. Müllen, and R. H. Friend, Phys. Rev. B 64, 125211 (2001).

${ }^{31}$ C. Gadermaier and G. Lanzani, J. Phys.: Condens. Matter 14, 9785 (2002).
${ }^{32}$ S. Backus, C. G. Durfee III, M. M. Murnane, and H. C. Kapteyn, Rev. Sci. Instrum. 69, 1207 (1998).

${ }^{33}$ P. Jonkheijm, F. J. M. Hoeben, A. P. H. J. Schenning, and E. W. Meijer (unpublished).

${ }^{34}$ D. Beljonne (unpublished).

${ }^{35}$ V. I. Klimov, D. W. McBranch, N. Barashkov, and J. Ferraris, Phys. Rev. B 58, 7654 (1998).

${ }^{36}$ D. W. McBranch, J. Kraabel, S. Xu, R. S. Kohlman, V. I. Klimov, D. D. C. Bradley, B. R. Hsieh, and M. Rubner, Synth. Met. 101, 291 (1999).

${ }^{37}$ B. Kraabel, V. I. Klimov, R. Köhlman, S. Xu, H.-L. Wang, and D. W. McBranch, Phys. Rev. B 61, 8501 (2000).

${ }^{38}$ R. C. Powell and Z. G. Soos, J. Lumin. 11, 1 (1975).

${ }^{39}$ T. Förster, Z. Naturforsch. A 4, 321 (1949).

${ }^{40}$ K. B. Eisenthal and S. Siegel, J. Chem. Phys. 41, 652 (1964).

${ }^{41}$ B. Y. Balagurov and V. G. Vaks, Sov. Phys. JETP 38, 968 (1974).

${ }^{42}$ B. Movaghar, M. Grünewald, B. Ries, H. Bässler, and D. Würtz, Phys. Rev. B 33, 5545 (1986).

${ }^{43}$ B. Mollay, U. Lemmer, R. Kersting, R. F. Mahrt, H. Kurz, H. F. Kauffmann, and H. Bässler, Phys. Rev. B 50, 10769 (1994).

${ }^{44}$ M. Yan, L. J. Rothberg, F. Papadimitrakopoulos, M. E. Galvin, and T. M. Miller, Phys. Rev. Lett. 73, 744 (1994).

${ }^{45}$ L. M. Herz and R. T. Phillips, Phys. Rev. B 61, 13691 (2000).

${ }^{46}$ G. Cerullo, M. Nisoli, S. Stagira, S. D. Silvestri, G. Lanzani, W. Graupner, E. List, and G. Leising, Chem. Phys. Lett. 288, 561 (1998).

${ }^{47}$ A. Suna, Phys. Rev. B 1, 1716 (1970).

${ }^{48}$ F. J. M. Hoeben, L. M. Herz, C. Daniel, P. Jonkheijm, A. P. H. J. Schenning, C. Silva, S. C. J. Meskers, R. T. Phillips, R. H. Friend, and E. W. Meijer (unpublished).

${ }^{49}$ F. C. Spano, J. Chem. Phys. 116, 5877 (2002).

${ }^{50}$ G. D. Scholes, X. J. Jordanides, and G. R. Fleming, J. Phys. Chem. B 105, 1640 (2001).

${ }^{51}$ G. D. Scholes, Chem. Phys. 275, 373 (2002). 